\title{
Performance Analysis for Efficient Cluster Head Selection in Wireless Sensor Network Using RBFO and Hybrid BFO-BSO
}

\author{
A. Rajagopal ${ }^{1}$, S. Somasundaram ${ }^{1}$, B. Sowmya ${ }^{2}$ \\ ${ }^{1}$ Department of Electronics \& Instrumentation Engineering, Annamalai University, Chidambaram, India \\ ${ }^{2}$ Department of Electronics \& Communication Engineering, Alpha College of Engineering, Chennai, India
}

Email address:

rajagopalayyasamy@gmail.com (A. Rajagopal)

\section{To cite this article:}

A. Rajagopal, S. Somasundaram, B. Sowmya. Performance Analysis for Efficient Cluster Head Selection in Wireless Sensor Network Using RBFO and Hybrid BFO-BSO. International Journal of Wireless Communications and Mobile Computing. Vol. 6, No. 1, 2018, pp. 1-9. doi: 10.11648/j.wcmc.20180601.11

Received: November 18, 2017; Accepted: November 30, 2017; Published: January 26, 2018

\begin{abstract}
Wireless Sensor Network involves in the communication task which demands the devices to form a connected network for collecting and disseminating information through radio transmission. The main objective of the Wireless Sensor Network is to extend the network lifetime in the operational environment, to charge or to exchange the sensor node batteries is probably an impossible/unfeasible activity. The clustered network aims to select $\mathrm{CHs}$ that minimize transmission costs and energy. To maximize the network lifetime, optimal $\mathrm{CH}$ selection is important. Selections of $\mathrm{CH}$ are Non deterministic Polynomial (NP) hard. Recently natural swarm inspired algorithms like Particle Swarm Optimization (PSO) and Ant Colony Optimization (ACO) have found their way into this domain and proved their effectiveness. In this work the BFO is adapted for cluster head selection so that multiple objectives like reduced packet delivery ratio, improved cluster formation, improved network life time and reduced end to end delay are achieved. Also a novel Hybrid algorithm using Bacterial foraging Optimization (BFO) - Bee swarm Optimization (BSO) is attempted to analysis the number of clustered formed, end to end delay, packet drop ratio and lifetime.
\end{abstract}

Keywords: Wireless Sensor Network, Cluster Head Selection, Bacterial Forging Optimization (BFO), Bee Swarm Optimization (BSO), Particle Swarm Optimization (PSO)

\section{Introduction}

Wireless Sensor Network (WSN) is the emerging paradigm of ad-hoc wireless networks. This network consists of hundreds or even thousands of autonomous devices, the so called sensor nodes. The sensor node is a low-power constrained device, generally composed by, a processor with limited processing power, a restricted quantity of memory, a sensor board, which may contain different kinds of sensing device, a battery, which provides power for the sensor node and a radio for wireless communication. Sensor nodes are generally powered by battery and when organized are unattended for long period of time and is supposed to function typically for about couple of months to years. Therefore, energy is an inadequate resource in WSN and therefore it is an effective usage is critical to extend the entire sensor network lifetime.

An effective multi-level cluster algorithm using link correlation for heterogeneous WSN were adopted as level-k cluster heads and implementing network coding on those nodes increases network lifetime significantly and improve the energy efficiency [1]. Simulation of [2] shows that the new protocol could balance energy consumption and extend the time during which there is no node using up energy. The concept of associate cluster head selection based on the distance between the cluster and the base station and on the residual energy of the sensor node in wireless sensor network reduces the overhead of clustering process, reduces the load over cluster head, avoiding re-clustering and hence reduce the energy consumption within the cluster in large-scale and 
dense sensor networks [3]. A Connection-Constrained WSN (CCWSN) for multi-hop WSN with a connection bound for each sensor nodes (SN) controls the diameter of multi-hop clusters so as to derive the appropriate cluster size and increase network lifetime. This static clustering method does not require re-configuration which would entail additional energy consumption and time overhead [4].

The energy consumption of LEACH routing protocols for WSN system evaluated by performing simulations using the software Network Simulator 2 (NS-2), show the number of clusters affects the level of energy consumption [5]. The simulation results of the cluster based routing protocol indicated that the method leads to efficient transmission of data packets with less energy and therefore increased the network longevity as compared to LEACH-C and LEACH [6]. The two distance-based clustering routing protocols called DB-LEACH and DBEA-LEACH based on LEACH protocols selects a cluster head node by considering the geometric distance between the candidate nodes to the base station and also exams the residual energy of the node greater than the average residual energy level of nodes in the network [7].

A version of LEACH protocol called Optimized LEACH (OP-LEACH) is presented in [8] aims to reduce energy consumption within the wireless sensor network. Both existing LEACH and proposed OP-LEACH are evaluated through extensive simulations using OMNET++ simulator which showed that Op-LEACH performed better than LEACH protocol. A MATLAB based Simulation results show the $\mathrm{LEACH}$ heterogeneous environment significantly reduced energy consumption and increased the total lifetime of the WSN than LEACH homogeneous environment [9]. A homogeneous fuzzy logic based clustering algorithm with the sensor nodes have been hexagonally deployed in the homogeneous environment. This covers the sensing area efficiently along with reducing the redundancy in the data being transmitted by the sensor nodes [10].

A hybrid clustering algorithm which has two folds: Use the K-Means unsupervised learning algorithms to select the sensors belonging to each cluster using an arbitrary number of clusters and uses Particle Swarm Optimization (PSO) and Genetic Algorithms (GA) separately to select the best CHs were tested over number of experiments with various layouts. KPSO provided better results compared to the KGA [11]. A Fuzzy and Ant Colony Optimization (ACO) based MAC/Routing Cross-Layer Protocol (FAMACRO) for Wireless Sensor Networks that encompasses cluster head selection, clustering and inter-cluster routing protocols is compared with Distributed Energy Efficient Hierarchical Clustering, Unequal Hybrid Energy Efficient Distributed Clustering, Energy Efficient Unequal Clustering and Improved Fuzzy Unequal Clustering protocol shows that FAMACRO is $82 \%$ more energy efficient, has $5 \%$ to $30 \%$ more network lifetime and sends 91\% more packets compared to Improved Fuzzy Unequal Clustering protocol [12].
An intelligent method of routing based on particle swarm optimization algorithm with the objectives of optimization of sensor network lifetime with respect to the energy level of each node, routs costs and the importance of nodes in routing process [13]. An optimized hybrid clustering algorithm based on Honey Bee Mating algorithm and K-means resolve the drawbacks like complex optimization problem and poor convergence behavior [14]. The foraging optimization for $\mathrm{CH}$ Selection by which an improved $\mathrm{CH}$ selection for efficient data aggregation in sensor networks is realized with energy saving objective function has the high average throughput, low delay in seconds and low DATA dropping compared to LEACH [15].

The foraging optimization for cluster head selection is presented to obtain efficient data aggregation in sensor networks. The algorithm is based on BFO incorporated LEACH with energy saving objective function. The suggested BFO algorithm shows better performance in terms of selection of the number of clusters formed, lower average end to end delay $(\mathrm{sec})$, lower average packet drop ratio and increased lifetime of the network [16].

The $\mathrm{CH}$ selection is optimized using a hybrid algorithm based on the BFO and BSO is proposed. BFO tends to get in the local optimums. To overcome this, BFO is hybridized with $\mathrm{BSO}$ in a pipeline fashion. BSO is a popular technique to solve WSN optimization problems due to its simplicity, high solution quality, fast convergence and less computational burden. The proposed hybrid BFO-BSO algorithm shows better performance in terms of selection of the number of clusters formation, lower average end to end delay (sec), lower average packet drop ratio and increased lifetime of the network [17].

In this paper, an improved cluster head selection algorithm based on soft computing is proposed to obtain an efficient sensor networks data aggregation. The parameter evaluations are compared and investigated with the different algorithms like LEACH, KBFO, Refined-BFO (RBFO) algorithm and Hybrid BFO-BSO.

\section{Refined Bacterial Foraging Optimization (RBFO)}

In RBFO, bacterial foraging algorithm is used to increase the energy efficiency of every sensor node and it is employed in WSN. Bacterial foraging technique is gaining control optimization issues due to,

1. Biology provides extremely automatic, strong and effective organism.

2. Like a common fish, search strategy of bacterium is good.

3. Bacterium sense, create a choice and adopt social hunting.

In recent years, a population-based numerical optimization algorithm BFO provides rich source of solution in many engineering applications and computational model. BFO is applied to solve practical engineering problems like optimal 
control, harmonic estimation channel equalization and improved energy efficiency is provided by BFO for cluster head selection.

Based on the study of the bacterial foraging behaviours, Bacterial Foraging Optimization Algorithm (BFOA) is a well computational methodology. E. Coli bacterium alternates between running and tumbling.

E. Coli will swim longer if it swims up nutrient gradient where E. Coli will search again to avoid unfavorable environments when it swims down nutrient gradient. If all bacteria are killed in a region or a group is dispersed into a new part of the environment, event can occur. Effect of possibly destroying chemotactic progress and assisting to place bacteria near good food sources are presented in elimination and dispersal events.

Natural selection process eliminates an animal with poor foraging strategies and propagation of genes of those animals is preferred for successful foraging strategies since they are more likely to enjoy reproductive success. Poor foraging strategies are eliminated or shaped into good ones after many generations.

BFO, an Evolutionary based algorithm is a stochastic search methods that mimic the metaphor of natural biological evolution and it is operated on a population of potential solutions applying the principle of survival of the fittest to produce better and better approximations to a solution.

Based on fitness level in the problem domain, a new set of approximations is created at each generation by the process of selecting individuals. Breeds them together using operators borrowed from natural genetics. This process leads to the evolution of populations of individuals are better suited to their environment than the individuals that they were created.

Bacterial Foraging Algorithm (BFA) model is the behaviour of $\mathrm{E}$. Coli bacteria which have two types of movements such as swimming and tumbling. In swimming movement, a bacterium moves in a straight line in a given direction where tumbling changes its direction of movement randomly.

Three steps of BFA:

a) Chemo taxis step

b) Reproduction step

c) Elimination step

With random position, a bacterium is started initially. Movement directions are directed by evaluating the objective function. Again it moves in a particular direction based on the objective function evaluation. It moves in same direction if the move is better than previous one till it gets bad objective function. This process is called as chemo taxis. Reproduction occurs after specific number of chemo taxis steps to form a healthy bacterium by combining two bacteria. In elimination-dispersal step, a bacterium splits into two and a non-healthy bacterium is eliminated in this step.

The bacterium's orientation is modified by tumble. It moves in its current direction, during swimming-the chemo taxis step. An animal with poor foraging strategies is eliminated by the natural selection process and with successful foraging strategies it favors propagation of genes which are likely to have reproductive success. Poor foraging strategies are eliminated or converted to good one after generations.

Researchers use an optimization procedure. Till reduction in nutrient density, bacteria move several steps in the same direction and then tumble to a new direction. Adaptive step length strategy is adopted in chemotaxis step to adjust the bacteria's step size and this is average distance from neighbors. Angle of rotation is no longer random but maximum value of lure-degree that is ratio of node's residual energy and transmission energy consumption.

CHEMOTAXIS: Bacteria move and search for nutrient to maximize the energy level is named as Swimming and tumbling are the two modes of movement for the life time of a bacterium to perform in search of nutrient and it is represented as in the equation (1)

$$
\theta_{(j+1, k, l)}^{i}=\theta_{(j, k, l)}^{i}+c(i) *\left(\frac{\Delta(i)}{\sqrt{\Delta^{T}(i) \Delta(i)}}\right)
$$

This represents the chemotactic movement of the bacteria, where $\theta_{(j, k, l)}^{i}$ is represented by ith bacterium at $j$ th chemotactic, kth reproduction and lth elimination-dispersal steps. c (i) means the size of the steps taken at random direction and indicates a vector in the arbitrary direction whose element lies between $[-1,1]$.

Till a bacterium reaches a positive-nutrient gradient, chemotaxis movement continues. Best half of population undergoes reproduction eliminating others after specific number of swims. Local optima escape is ensured by an elimination-dispersion event where some bacteria are settled randomly with a small probability and new replacements initialized at random search space locations. Figure 1 shows the flowchart of a RBFO algorithm.

Novel objective function of Refined BFO (RBFO) is proposed as given by equation (2):

$$
\min f_{i}(x)=\alpha_{1}(\min (P L R))+\alpha_{2}\left(\min \left(\frac{E_{i}^{r}}{E_{\text {initial }}}\right)\right)
$$

Where $\min f_{i}(x)$ is the minimum objective function of cluster head selection

$\mathrm{E}_{\text {initial }}$ is the initial energy in the node

$E_{i}^{r}$ is the remaining energy in node $I$

PLR is the Packet Loss Ratio, $\alpha_{1}$ and $\alpha_{2}$ is the scaling factors.

Some actions of Chemotaxis are:

a. In neutral medium, alternate tumbles and runs $\Rightarrow$ search.

b. If swimming up a gradient nutrient or out of noxious substances, swim longer when climb up nutrient gradient or down noxious gradient $\Rightarrow$ seek increasingly favorable environments.

c. If swimming down gradient nutrient or up noxious substance gradient, then search $\Rightarrow$ to avoid unfavorable environments.

REPRODUCTION: First half of bacteria population 
survives where the remaining bacteria split into two which are the placed in the same position as their parent. Fitness value for the ith bacterium during its life after $\mathrm{N}_{\mathrm{c}}$ Steps can be representing as in equation (3):

$$
J_{\text {health }}^{i}=\sum_{j=1}^{\left(N_{C}+1\right)} j_{(j, k, l)}
$$

$J_{\text {health }}^{i}$ means the health of bacteria, sort the bacteria in ascending values. So the $\mathrm{x}^{\text {th }}$ bacteria with highest $J_{\text {health }}^{i}$ values die and other $x^{\text {th }}$ bacteria with the best values split into two. Therefore reproduction step keeps the bacteria ratio constant.

ELIMINATION AND DISPERSAL: BFO algorithm makes some bacteria to get eliminated and dispersed with probability $\mathrm{P}_{\text {ed }}$ after $\mathrm{N}_{\mathrm{re}}$ number of reproductive events by ensuring that the bacteria do not get trapped into a local optimum instead of the global optima Dhiman (2013).

Bacteria swarm $\mathrm{S}$ behaves as in the following steps Mezura-Montes and Hernández-Ocana (2008):

1. In nutrient's map, bacteria are randomly distributed.

2. In a map, bacteria moves to high-nutrient regions and those in noxious substance regions or low-nutrient regions die/disperse. It is in convenient regions reproduce (split).

3. In favorable regions of nutrients map, bacteria are located and it tried to attract other bacteria by generating chemical attractants.

4. At highest-nutrient region, bacteria are now located.

5. Then disperse to look for new nutrient regions in a map.

The procedures implemented are:

$\mathrm{f}$ is the objective function of cluster head selection as shown in equation (4):

$$
f=\beta \cdot f_{1}+(1-\beta) \cdot f_{2}
$$

Where $f_{1}$ is the nodes maximum average Euclidean distance of nodes to their associated cluster heads, $f_{2}$ is the ratio of total initial energy of all nodes to the total energy of the cluster head candidates, $\beta$ means the scaling factor indicates the contribution of $f_{1}$ and $f_{2} f_{1}$ and $f_{2}$ are represented as in equations (5) and (6):

$$
\begin{gathered}
f_{1}=M A X_{k=1.2 .3, \ldots k}\left\{\sum_{\forall n_{i} \in c_{p, k}} \frac{d\left(n_{i}, C H_{p, k}\right)}{\left|C_{p, k}\right|}\right\} \\
f_{2}=\frac{\sum_{i=1}^{N} E\left(n_{i}\right)}{\sum_{i=1}^{N} E\left(C_{p, k}\right)}
\end{gathered}
$$

Where $E\left(n_{i}\right)$ the total initial energy of all nodes, $E\left(C_{p, k}\right)$ is the total energy of cluster head candidates. Here, $\mathrm{N}$ is number of nodes of which $\mathrm{K}$ is elected as CHs. $\left|\mathrm{C}_{\mathrm{p}, \mathrm{k}}\right|$ are nodes that belong to cluster $C_{k}$ in particle $p$, ensures that only nodes with above average energy resources are elected as $\mathrm{CHs}$, with minimum average distance between nodes and CHs.

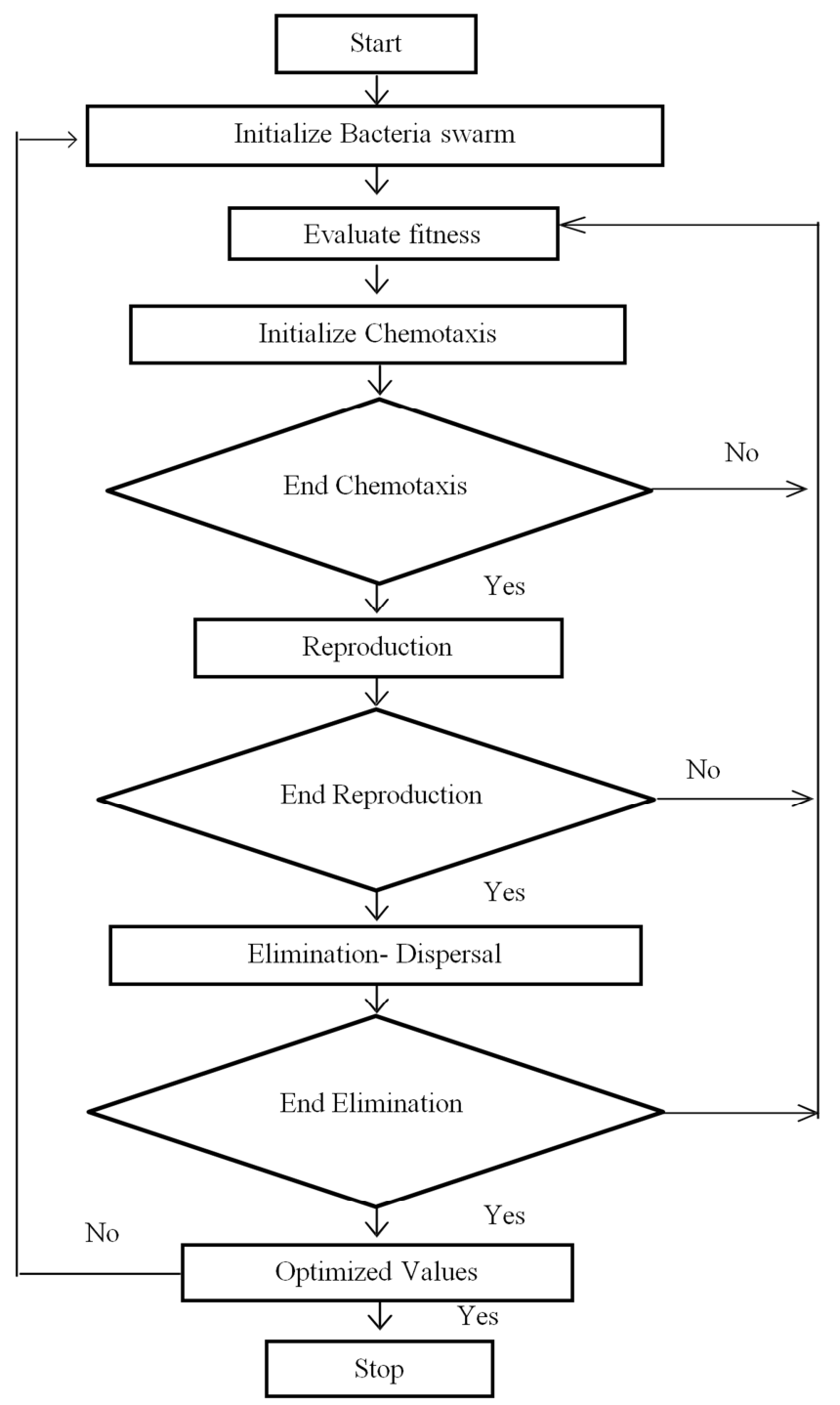

Figure 1. Flowchart for the RBFO.

\section{Hybrid BFO-BSO}

Hybridization approach is used to enhance capability of optimization algorithms by reducing computational cost by making a simple and better structure to lead to higher performance. With rapidly emerging computing tools and efficiency in current technology, hybrid approaches have become increasingly popular to explore. Researchers consider various combinations of optimization algorithms to increase system performance.

Hybrid optimization is a new class of optimization heuristics which assume that one has implemented two or more algorithms for the same optimization. It chooses best one and to construct a hybrid register allocator between two different register allocation algorithms, graph coloring and linear scan are used Cavazos et al., (2006). It aims at creating an allocator that achieves a good balance between two factors such as to find a good packing of the variables to registers and to reduce the overhead of the allocator. Hybrid optimization reduce compilation effort, using an efficient algorithm most of the time, but use a more effective, but 
expensive, optimization algorithm seldom, when it deems the additional benefit is worth the effort.

New class of biologically stochastic global search techniques includes BFO which mimics E. coli bacteria's foraging behaviour. Food is located, handled and ingested by this method. Bacterium shows tumbling or swimming actions in foraging process. Till bacterium goes to a positive-nutrient gradient, the movement of chemotaxis continues.
Population's best half undergoes reproduction process once a specific complete swim is done by eliminating the reminder of the population. The elimination-dispersion event escapes local optima and randomly some of the bacteria are killed with a very small probability. At random search space locations, new replacements are initialized.

In section II, equations 1 to 6 are cited. RBFO is used to obtain an objective function of Hybrid BFO-BSO.

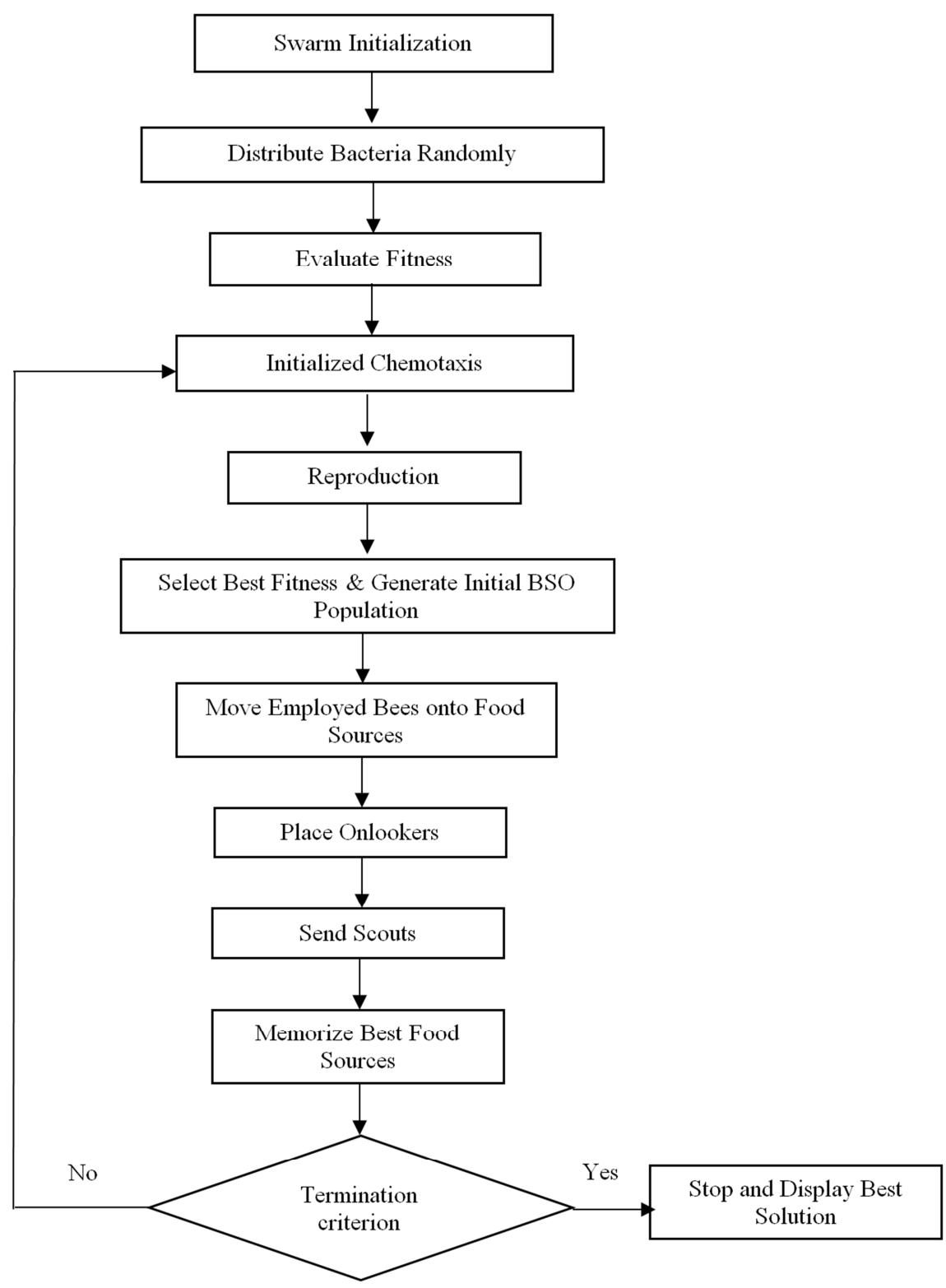

Figure 2. Flowchart for Hybrid BFO-BSO.

BFO has local optima and it is hybridized with Bee Swarm Optimization (BSO) to overcome the optima problem. A bacterium gets mutated by a BSO operator once a chemotactic step is experienced in new approach. Bacterium is attracted to a globally best position which is found in entire population till then at the current time and toward its earlier heading direction.

In a pipeline, both $\mathrm{BFO}$ and $\mathrm{BSO}$ algorithms are run with the output of each being supplied as input to the next algorithm. Study on foraging strategies of honey bee colonies were done by Seeley (1995). Common features such as to distribute food collection, individuals having limited abilities and indirect communication between individuals are shared by both Honey bee and ant colonies.

Their foraging processes differ because insect's nature is differed between the two types of colonies with foraging ants moving on a terrain and bees flying. Bees leave the hive during foraging process and search for new nectar sources. Nectars are brought back if it is found to the hive and to exploit the food site by competing with each other during recruitment other bees are recruited. 
In BSO, a colony of artificial bees consists of three bee groups such as employed bees, onlookers, and scouts. Onlooker is the bee waiting for the dance area to decide on a food source and that going to it is the employed bee. Scout bee carries random search to discover new sources. Optimization problem solution is represented by the food source position and nectar in a food source corresponds to the associated solution's quality calculated by equation (7):

$$
f i t_{i}=\frac{1}{1+f_{i}}
$$

Food source is choosing based on the probability value associated with it, $\mathrm{p}_{\mathrm{i}}$, an artificial onlooker is calculated by equation (8):

$$
p_{i}=\frac{f_{i t_{i}}}{\sum_{n=1}^{S N} f i t_{n}}
$$

Where $\mathrm{SN}$ is number of food sources which is equal to the number of employed bees, and $f i t_{i}$ means the fitness of the solution which is inversely proportional to the $f_{i}$ where $f_{i}$ means the cost function of the clustering problem.

\section{Results and Discussions}

The present work is to enhance the network lifetime through improved cluster head selection. The performance metrics used in the study include the number of clusters formed, the end to end delay, packet drop ratio and the lifetime (number of alive nodes against number of rounds).

The simulations are conducted by using MATLAB software tool for varying number of nodes with a single base station in a $2 \mathrm{sq}$. $\mathrm{km}$ area. The number of nodes in a network ranges from 30 to 1200 . The performances of clustering protocols for WSN are evaluated. The simulation results of the present work viz. Hybrid BFO-BSO, RBFO are compared with the early works viz. KBFO and LEACH.

A. PERFORMANCE MEASURES \&RESULTS FOR NETWORK SIZE-(30 to 1200) NODES

After every simulation the number of nodes is incremented in steps of 30up to 200 and after 200 it is incremented by 200. A total of the 48 different simulation setup are carried out as shown in Table 1. The comparisons are made for different network sizes. The performances of cluster head selection in protocols for WSN are evaluated.

Table 1. Protocols Used in the study.
The simulation parameters used for the study to Hybrid, RBFO, KBFO, LEACH and their values are depicted in Table 2.

Table 2. Simulation details used for the study.

\begin{tabular}{ll}
\hline Parameter used & Value used \\
\hline $\boldsymbol{\Delta}(\mathrm{i})$ & {$[-1,1]$} \\
Number of Bacteria in colony S & 50 \\
Swim steps Ns & 4 \\
Chemotactic step Nc & 100 \\
$\mathrm{~Np}$ & 4 \\
$\mathrm{C}_{\text {initial }}$ & 0.1 \\
$\mathrm{~A}$ & 10 \\
\hline
\end{tabular}

After carrying out simulations for the different protocols, the results for the number of clusters formed is tabulated in Table 3 and Table 4 describes the improvement obtained. The output for the number of clusters formed is graphically shown in Figure 3.

\section{B. NUMBER OF CLUSTERS FORMED}

The cluster formation procedures should generate the best possible clusters that are well balanced. The cluster formation can schedule activities in the cluster so that nodes can switch to the low-power sleep mode and reduce the energy consumption. The sensors are engaged in a round robin order and the time of their transmission and reception can be determined so that the sensor retries are avoided, redundancy in coverage can be limited. It is an important performance metrics to reduce the sensor energy by increasing the lifetime through selecting the cluster heads. However, they should also preserve the number of exchanging messages low and the total time complexity should (if possible) remain constant and independent of the growth of the network.

\section{I) NUMBER OF CLUSTERS}

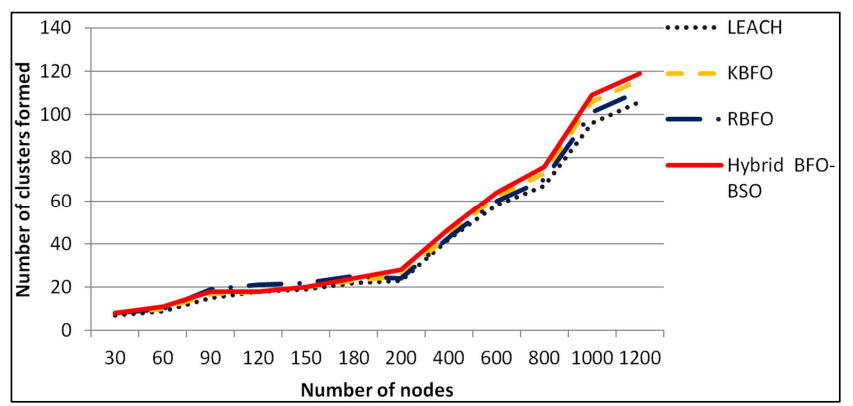

Figure 3. Number of clusters formed.

\begin{tabular}{ll}
\hline Number of nodes & $30,60,90,120,150,180,200,400,600,800,1000,1200$ \\
Protocols used & LEACH, KBFO, Refined BFO, Hybrid BFO-BSO \\
\hline
\end{tabular}

Table 3. Number of Clusters Formed.

\begin{tabular}{lllll}
\hline Number of nodes & Protocols & KEACH & RBFO & Hybrid BFO-BSO \\
\hline 30 & 7 & 8 & 8 & 10 \\
60 & 9 & 10 & 19 & 11 \\
90 & 15 & 17 & 21 & 18 \\
120 & 18 & 18 & 22 & 20 \\
150 & 19 & 20 & 23 & 24 \\
180 & 22 & 23 & 24 & 28 \\
200 & 23 & 25 & & \\
\hline
\end{tabular}




\begin{tabular}{|c|c|c|c|c|}
\hline $\begin{array}{l}\text { Protocols } \\
\text { Number of nodes } \\
\end{array}$ & LEACH & KBFO & RBFO & Hybrid BFO-BSO \\
\hline 400 & 42 & 44 & 43 & 47 \\
\hline 600 & 58 & 62 & 60 & 64 \\
\hline 800 & 67 & 73 & 70 & 76 \\
\hline 1000 & 96 & 106 & 101 & 109 \\
\hline 1200 & 106 & 116 & 111 & 119 \\
\hline
\end{tabular}

\section{END TO END DELAY (S)}

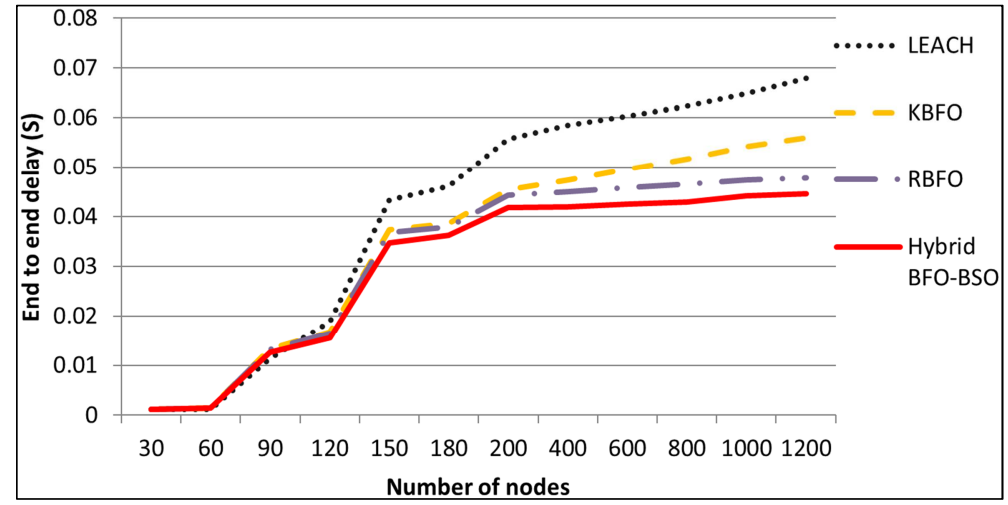

Figure 4. End to end delay (S).

Table 4. End to end delay (S).

\begin{tabular}{|c|c|c|c|c|}
\hline $\begin{array}{l}\text { Protocols } \\
\text { Number of nodes }\end{array}$ & LEACH & KBFO & RBFO & Hybrid BFO-BSO \\
\hline 30 & 0.001242 & 0.001196 & 0.001183 & 0.001115 \\
\hline 60 & 0.001138 & 0.001505 & 0.00149 & 0.001393 \\
\hline 90 & 0.011368 & 0.013278 & 0.013081 & 0.012633 \\
\hline 120 & 0.018795 & 0.016731 & 0.016424 & 0.015573 \\
\hline 150 & 0.043275 & 0.037391 & 0.036754 & 0.034752 \\
\hline 180 & 0.04613 & 0.038646 & 0.037921 & 0.03626 \\
\hline 200 & 0.055686 & 0.045392 & 0.044341 & 0.041769 \\
\hline 400 & 0.058415 & 0.047319 & 0.044971 & 0.041896 \\
\hline 600 & 0.060306 & 0.049649 & 0.04589 & 0.042503 \\
\hline 800 & 0.062321 & 0.051701 & 0.046469 & 0.042896 \\
\hline 1000 & 0.064872 & 0.05416 & 0.047311 & 0.044223 \\
\hline 1200 & 0.067958 & 0.056029 & 0.047814 & 0.044515 \\
\hline
\end{tabular}

End-to-end delay refers to the time taken for a packet to be transmitted across a network from source to destination. Due to the nature of a WSN, delay guarantees achieved through providing deadlines to each individual message on the network and the application level.

\section{PACKET DROP RATIO}

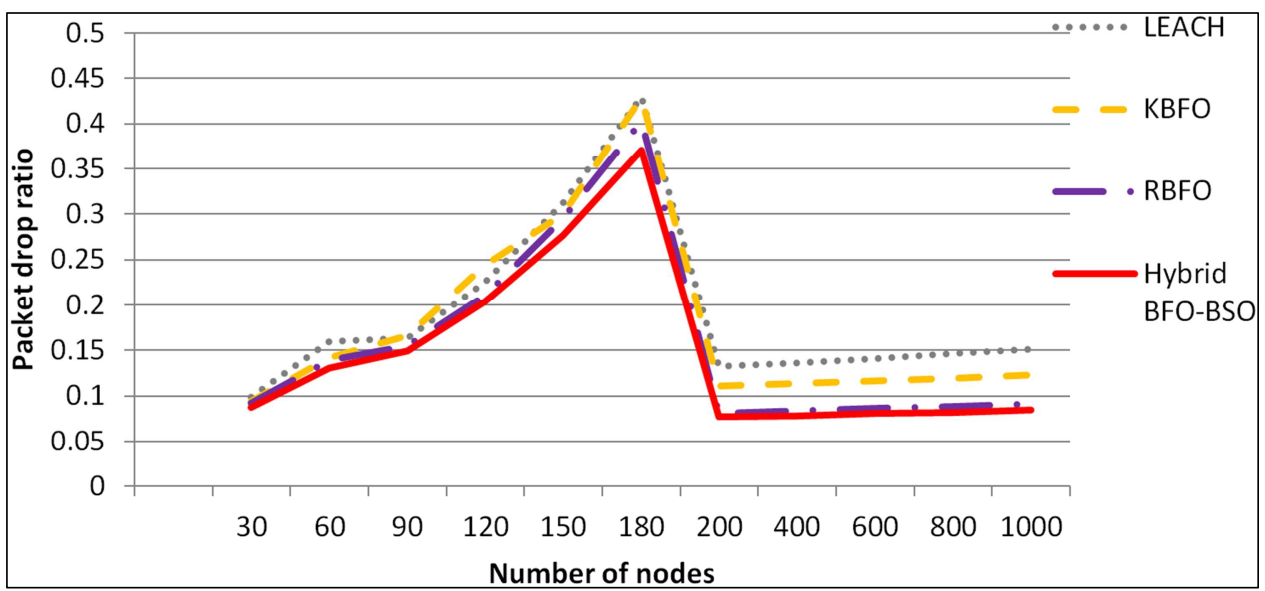

Figure 5. Packet drop ratio. 
Table 5. Packet drop ratio.

\begin{tabular}{|c|c|c|c|c|}
\hline Number of nodes & Protocols LEACH & KBFO & RBFO & Hybrid BFO-BSO \\
\hline 30 & 0.0985 & 0.0941 & 0.0922 & 0.0879 \\
\hline 60 & 0.16 & 0.1418 & 0.139 & 0.1305 \\
\hline 90 & 0.163 & 0.1663 & 0.1546 & 0.1492 \\
\hline 120 & 0.2252 & 0.2439 & 0.21 & 0.2036 \\
\hline 150 & 0.3129 & 0.3007 & 0.296 & 0.2765 \\
\hline 180 & 0.4295 & 0.4283 & 0.4028 & 0.3708 \\
\hline 200 & 0.1324 & 0.1112 & 0.0811 & 0.077 \\
\hline 400 & 0.1362 & 0.1137 & 0.0838 & 0.0781 \\
\hline 600 & 0.1409 & 0.1165 & 0.0861 & 0.0806 \\
\hline 800 & 0.1464 & 0.1195 & 0.0884 & 0.0819 \\
\hline 1000 & 0.1512 & 0.1231 & 0.091 & 0.0845 \\
\hline 1200 & 0.1566 & 0.126 & 0.0943 & 0.0884 \\
\hline
\end{tabular}

The Packet drop occurs when one or more packets of data travelling across a Wireless Sensor Network fail to reach their destination. Packet drop is typically caused by network congestion. Packet drop is measured as the ratio of packets lost with respect to the packets sent. This metric is used to detect the best delivery of packets sent to the network.

E. LIFETIME (ALIVE NODES)

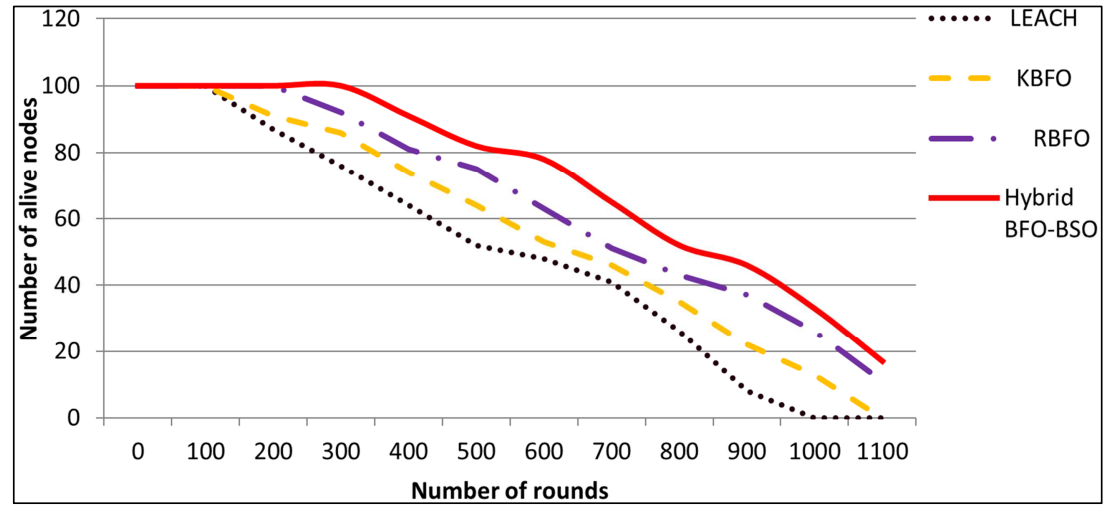

Figure 6. Lifetime (alive nodes).

Table 6. Lifetime (alive nodes).

\begin{tabular}{|c|c|c|c|c|}
\hline \begin{tabular}{|ll} 
Number of rounds & Protocols \\
\end{tabular} & LEACH & KBFO & RBFO & Hybrid BFO-BSO \\
\hline 0 & 100 & 100 & 100 & 100 \\
\hline 100 & 100 & 100 & 100 & 100 \\
\hline 200 & 87 & 91 & 100 & 100 \\
\hline 300 & 76 & 86 & 92 & 100 \\
\hline 400 & 64 & 74 & 81 & 91 \\
\hline 500 & 52 & 64 & 75 & 82 \\
\hline 700 & 41 & 46 & 51 & 65 \\
\hline 800 & 26 & 35 & 43 & 52 \\
\hline 900 & 8 & 22 & 37 & 46 \\
\hline 1000 & 0 & 13 & 26 & 33 \\
\hline 1100 & 0 & 0 & 11 & 17 \\
\hline
\end{tabular}

Lifetime is considered as one of the most important parameters to evaluate in Wireless Sensor Networks. Lifetime is defined as the time that the network can run fully operative. This is the time until the first node dies. The metric ensures the maximum per-node load, where the nodes load corresponds to the number of packets sent from or routed through the given node. Frequent recharging or replacements of batteries are warranted with too many nodes of the network and hostile environment. Hence lifetime improvement assumes importance in networks.

\section{Conclusion}

RBFO and Hybrid BFO-BSO based Cluster Head Selection for Wireless Sensor Network are performed and measured for the number of clusters formed, end to end delay, Packet drop ratio and lifetime. The comparison of RBFO and Hybrid BFO-BSO with KBFO and $\mathrm{LEACH}$ are listed for all performance measured in detail with graphically and literally. The optimization algorithm RBFO and Hybrid 
BFO-BSO gives the lifetime improvement and hence increases the battery life. The packet drop ratio, end to end delay is reduced in these optimization techniques. The parametric comparisons reveal that from the power of view of the overall performance, the order of superiority is Hybrid BFO-BSO, RBFO, KBFO and LEACH. Further work on Hybrid BFO-BSO that has been found to be the best in the present study may include testing in MANET environment.

\section{References}

[1] Praveena, N. G., and Prabha, H. (2014), An efficient multilevel clustering approach for a heterogeneous Wireless Sensor Network using link correlation, EURASIP Journal on Wireless Communications and Networking, Vol. 1, pp. 1-10.

[2] Li, J. A., Zhou, J., and Zhang, Y. (2014), Cluster Head Selection Based on an Information Factor for Wireless Sensor Network Protocol, Journal of Networks, Vol. 9, No. 9, pp. 2384-2391.

[3] Gupta, S. K., and Sinha, P. (2014), Overview of Wireless Sensor Network, A Survey, International Journal of Advanced Research in Computer and Communication Engineering, Vol. 3, No. 1, pp. 5201-5207.

[4] O. Chipara, Z. He, G. Xing, Q. Chen, X. Wang, C. Lu, J. Stankovic, T. Abdelzaher, (2006) Real-time power-aware routing in sensor networks, quality of service, in: IWQoS 2006, 14th IEEE International, Workshop, pp. 83-92.

[5] Mahyastuti, V. W., and Pramudita, A. A. (2013), Energy consumption evaluation of low energy adaptive clustering hierarchy routing protocol for wireless sensor network, In Communication, Networks and Satellite (COMNETSAT), IEEE International Conference, pp. 6-9.

[6] Tripathi, M., Battula, R. B., Gaur, M. S., and Laxmi, V. (2013), Energy Efficient Clustered Routing for Wireless Sensor Network, In Mobile Ad-hoc and Sensor Networks (MSN), IEEE Ninth International Conference, pp. 330-335.

[7] Nguyen, T. G., So-In, C., and Nguyen, N. G. (2014), Two energy-efficient cluster head selection techniques based on distance for Wireless Sensor Networks, In Computer Science and Engineering Conference (ICSEC), International, pp. 3338 .

[8] Gambhir, S., and Fatima, N. (2014), Op-LEACH: An
Optimized LEACH Method for Busty Traffic in WSNs, In Advanced Computing and Communication Technologies (ACCT), Fourth International Conference, pp. 222-229.

[9] Sujee, R., and Kannammal, K. E. (2015), Behavior of LEACH protocol in heterogeneous and homogeneous environment, In Computer Communication and Informatics (ICCCI), International Conference, pp. 1-8.

[10] Sharma, T., Tomar, G. S., Berry, I., Kapoor, A., and Jasuja, S. (2016), Cluster Head Election with Hexagonal Node Deployment Technique in Wireless Sensor Networks, International Journal of Future Generation Communication and Networking, Vol. 9, No. 1, pp. 247-258.

[11] Sheta, A., and Solaiman, B. (2015), Evolving a Hybrid KMeans Clustering Algorithm for Wireless Sensor Network Using PSO and GAs. International Journal of Computer Science Issues (IJCSI), Vol. 12, No. 1, pp. 23-32.

[12] Gajjar, S., Sarkar, M., and Dasgupta, K. (2015), FAMACRO: Fuzzy and ant colony optimization based MAC/routing crosslayer protocol for wireless sensor networks, Procedia Computer Science, Vol. 46, pp. 1014-1021.

[13] Ahmadi, R., and Masdari, M. (2015), providing an efficient algorithm for wireless sensor network routing with hybrid particle swarm optimization and LEACH. Academie Royale Des Sciences D Outre-Mer Bulletin Des Seances, Vol. 4, No. 3, pp. 80-88.

[14] Teimoury, E., Gholamian, M. R., Masoum, B., and Ghanavati, M. (2016), An optimized clustering algorithm based on Kmeans using Honey Bee Mating algorithm.

[15] Kavitha, G., and Wahidabanu, R. (2014 March), Foraging Optimization for Cluster Head Selection, Journal of Theoretical and Applied Information Technology, Vol. 61, No. 3, pp. 571.

[16] Rajagopal, A., Somasundaram, S., Sowmya, B. and Suguna, T. (2015), Soft computing based Cluster Head Selection in Wireless Sensor Network using Bacterial Foraging Optimization Algorithm, International Journal of Electrical, Computer, Energetic, Electronics and Communication Engineering, WASET, Vol. 9 (3), pp. 357-362.

[17] Rajagopal, A., Somasundaram, S., Sowmya, B. and Suguna, T. (2015), Cluster Head Selection in Wireless Sensor Network using Hybrid BFO-BSO Algorithm, International Journal of Applied Engineering Research, RI publications, Vol. 10 (17), pp. $38245-36250$. 